Pacific Journal of Mathematics

A DECOMPOSITION THEOREM FOR TOPOLOGICAL GROUP 


\title{
A DECOMPOSITION THEOREM FOR TOPOLOGICAL GROUP EXTENSIONS
}

\author{
ARNOLD J. INSEL
}

\begin{abstract}
G. W. Mackey has developed a characterization of the group of equivalence classes of extensions of a fixed group $G$ by a fixed abelian group $A$ restricting all groups to be locally compact second countable Hausdorff spaces. Calvin C. Moore incorporated his results into a cohomology theory of group extensions such that the second cohomology group, $H^{2}(G, A)$, coincides with Mackey's group of extension classes. The purpose of this paper is to consider the special case in which $G$ and $A$ are connected, $A$ is a lie group, $G$ is locally arcwise connected and locally simply connected. Under these conditions $G$ and $A$ admit universal covering groups $U_{G}$ and $U_{A}$. Allowing $\pi(G)$ and $\pi(A)$ to denote the fundamental groups of $G$ and $A$ respectively (with basepoint the identity) any extension of $G$ by $A$ determines an extension of $U_{G}$ by $U_{A}$ and an extension of $\pi(G)$ by $\pi(A)$ uniquely up to equivalence. Hence there is a map $\Phi$, in fact a homomorphism, constructed from $H^{2}(G, A)$ to $H^{2}\left(U_{G}, U_{A}\right) \oplus H^{2}(\pi(G), \pi(A))$. In this paper $H^{2}(G, A)$ is determined as a direct sum of subgroups of $H^{2}\left(U_{G}, U_{A}\right)$ and $H^{2}(\pi(G), \pi(A))$, and of a third group which is computed.
\end{abstract}

Throughout all topological groups are assumed to be locally compact, Hausdorff and second countable.

Let $G$ and $A$ be topological groups, $A$ abelian. $A$ is called a $G$ module if there is given an action of $G$ on $A$, that is, a continuous function from $G \times A$ into $A$, carrying $(g, a)$ onto $g a$, such that (i) for any fixed $g \in G$, the mapping of $A$ into $A$ determined by $a \longrightarrow g a$ is an automorphism, (ii) the automorphism thus determined by the identity of $G$ is the identity automorphism, and (iii) for any $g$ and $h$ in $G$ and $a \in A,(g h) a=g(h a)$. If $A$ is a $G$-module then an extension of $G$ by $A$ is an exact sequence

$$
D: 1 \longrightarrow A \stackrel{i}{\longrightarrow} E \stackrel{j}{\longrightarrow} G \longrightarrow 1
$$

such that $E$ is a topological group, $i: A \longrightarrow i(A)$ is a homeomorphism and $E / i(A)$ is topologically isomorphic to $G$ or equivalently, $j$ is open, and finally, the action of $G$ on $A$ defined by $x a=i^{-1}\left(x i(a) x^{-1}\right)$ coincides with the given action of $G$ on $A$. Sometimes, when $G$ and $A$ are fixed, and when no confusion can arise, the extension $D$ will be denoted by $D(E, i, j)$. Two extensions $D(E, i, j)$ and $D^{\prime}\left(E^{\prime}, i^{\prime}, j^{\prime}\right)$, of $G$ by $A$ are called equivalent if there is a topological isomorphism $\phi$ of 
$E$ onto $E^{\prime}$ such that $\phi i=i^{\prime}$ and $j^{\prime} \phi=j$. Let $\operatorname{Ext}(G, A)$ denote the family of equivalence classes of group extensions of $G$ by $A$. The problem of determining $\operatorname{Ext}(G, A)$ was essentially solved by G. W. Mackey in [7] by applying certain results on Borel groups proved in [6]. The solution involves the theory of analytic Borel spaces (c.f. the introduction of [1]). We shall assume the definitions and results of [1]. One useful result is that for the exact sequence

$$
D: 1 \longrightarrow A \stackrel{i}{\longrightarrow} E \stackrel{j}{\longrightarrow} G \longrightarrow 1
$$

to be an extension of $G$ by the $G$-module $A$, the requirements on $i$ and $j$ can be weakened to only require that $i$ and $j$ be continuous. One proves that $i$ is a homeomorphism of $A$ onto $i(A)$ and $j$ is open. In [10] C. C. Moore incorporates Mackey's solution to the extension problem into a cohomology theory of topological groups. In his theory the second cohomology group of $G$ with respect to $A, H^{2}(G, A)$, can be put into a one-to-one correspondence with $\operatorname{Ext}(G, A)$. We shall draw upon the results of [10] and identify $\operatorname{Ext}(G, A)$ with $H^{2}(G, A)$.

1. Construction of $\Phi$. We fix topological groups $G$ and $A$ so that $A$ is a connected abelian Lie group with an action of $G$, and $G$ is connected, locally arcwise connected, and locally simply connected. We fix an extension

$$
D: 1 \longrightarrow A \stackrel{i}{\longrightarrow} E \stackrel{j}{\longrightarrow} G \longrightarrow 1
$$

of $G$ by $A$.

Proposition 1.1. $j: E \rightarrow G$ is a locally trivial fibre space with fibre $A$.

Proof. $A$ is a product of a vector group $R^{m}$ and an $n$-torus $T^{n}$, and hence $A$ is a covering group for $T^{m+n}$. Hence the result easily follows from [3] and the fact that $A$ acts on $E$ as a group of transformations by $a(x)=i(\alpha) x$.

Proposition 1.2. $E$ is connected, locally arcwise connected and locally simply connected. Hence $E$ as well as $G$ and $A$ admit universal covering groups.

Proof. $E$ is connected since $A$ and $G$ are. By Proposition 1.1, $E$ is locally a product of $A$ and $G$. Thus the other two properties carry over from $A$ and $G$ to $E$. Thus $A, E$ and $G$ each more than satisfy the conditions required to construct covering groups (e.g., see [2]). 
Let $U_{A}, U_{E}$, and $U_{G}$ denote the respective universal covering groups of $A, E$, and $G$, with respective projections $p_{1}, p_{2}$, and $p_{3}$. It is well known that the kernel of each $p_{i}$ is a closed, countable, discrete, and central subgroup. Also the kernel of each $p_{\imath}$ can be considered the fundamental group of $A, E$, and $G$ respectively, and is therefore denoted by $\pi(A), \pi(E)$, and $\pi(G)$. Let $i_{1}, i_{2}$, and $i_{3}$ denote the inclusion maps of $\pi(A)$ into $U_{A}$, etc. Thus we obtain three exact sequences

$$
V_{1}\left(U_{A}, i_{1}, p_{1}\right), V_{2}\left(U_{E}, i_{2}, p_{2}\right) \text { and } V_{3}\left(U_{G}, i_{3}, p_{3}\right) \text {. }
$$

Each $V_{i}$ is an extension of the appropriate group by the appropriate fundamental group, with trivial group action. Since $U_{A}, U_{E}$, and $U_{G}$ are simply connected, the homomorphisms $i$ and $j$ lift to unique homomorphisms $\tilde{i}$ from $U_{A}$ to $U_{E}$ and $\tilde{j}$ from $U_{E}$ to $U_{G}$ such that $p_{2} \tilde{i}=i p_{1}$, and $p_{3} \widetilde{j}=j p_{2}$. It is easily seen that $\tilde{i}$ maps the kernel of $p_{1}$ into the kernel of $p_{2}$ and that $\widetilde{j}$ maps the kernel of $p_{2}$ into the kernel of $p_{3}$, yielding homomorphisms $i_{\sharp}$ and $j_{\#}$ with $i_{2} i_{\#}=\widetilde{i} i_{1}$ and $i_{3} j_{\ddagger}=\widetilde{j} i_{2}$. Thus we obtain a 3 by 3 commutative diagram.

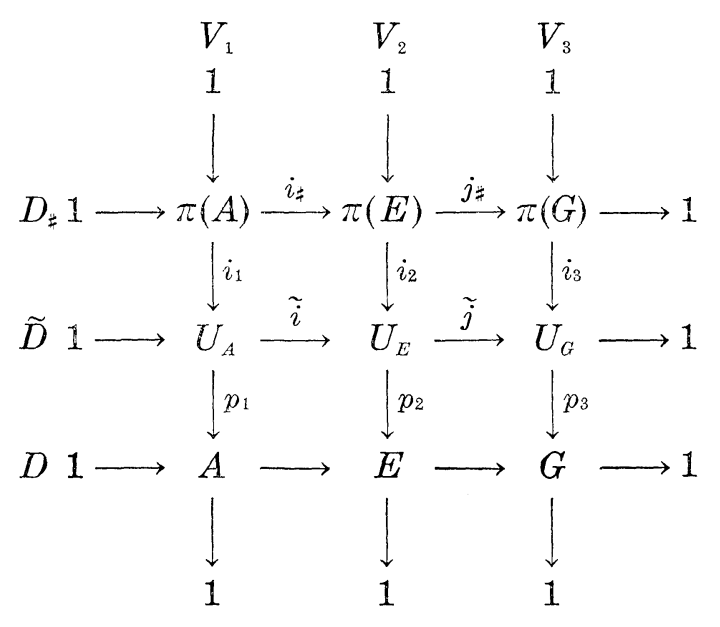

Diagram 1.

Let $D_{\#}$ denote the top row and $\widetilde{D}$, the middle row of Diagram 1 . Of course $D, V_{1}, V_{2}$, and $V_{3}$ are exact sequences.

Proposition 1.3. (1) $D_{\#}$ and $\widetilde{D}$ are exact sequences.

(2) The induced action of $U_{G}$ on $U_{A}$ is compatible with the given action of $G$ on $A$. That is, for any $x \in U_{G}$ and $t \in U_{A}$,

$$
p_{1}(x t)=\left(p_{3}(x)\right)\left(p_{1}(t)\right) \text {. }
$$

Furthermore this action is the only action of $U_{G}$ on $U_{A}$ compatible with the action of $G$ on $A$. 
( 3 ) $D_{\#}$ and $D$ are extensions of $U_{G}$ by $U_{A}$ and $\pi(G)$ by $\pi(A)$ respectively, with the action of (2), of $U_{G}$ on $U_{A}$, and trivial action of $\pi(G)$ on $\pi(A)$.

Proof. First note that, by the construction of the universal covering groups, $i_{\#}$ and $j_{\sharp}$ are actually the standard morphisms induced by the functor $\pi=\pi_{1}$, operating on $i$ and $j$. By Proposition $1.1 j$ : $E \rightarrow G$ is a fibre space with fibre $A$ and hence we obtain the exact homotopopy sequence of the fibering (see [5], p. 152):

$$
\cdots \longrightarrow \pi_{2}(G) \stackrel{d_{\sharp}}{\longrightarrow} \pi_{1}(A) \stackrel{i_{\sharp}}{\longrightarrow} \pi_{1}(E) \stackrel{j_{\sharp}}{\longrightarrow} \pi_{1}(G) \stackrel{d_{\sharp}}{\longrightarrow} \pi_{0}(A) .
$$

Since $G$ is an inverse limit of Lie groups (e.g., see [9]) by [11], $\pi_{2}(G)=0$. Since $A$ is connected, $\pi_{0}(A)=0$. Hence $D_{\sharp}$ is exact. Exactness of $\widetilde{D}$ follows by standard diagram chasing (see [8], p. 51); the needed condition $\widetilde{j} \widetilde{i}$ is trivial holds, since $\tilde{j} \widetilde{i}$ maps the connected group $U_{A}$ into the discrete group $i_{3}(\pi(G))$.

The first part of (2) follows simply from the commutativity of diagram 1. For the second part let $\alpha$ and $\beta$ be two actions of $U_{G}$ on $U_{A}$ compatible with the action of $G$ on $A$. Then for any $x \in U_{G}$ and $t \in U_{A}, \quad p_{1}(\alpha(x, t))=p_{1}(\beta(x, t))=\left(p_{3}(x)\right)\left(p_{1}(t)\right)$. Define a function $F$ from $U_{G} \times U_{A}$ to $U_{A}$ by $F(x, t)=\alpha(x, t)(\beta(x, t))^{-1}$. Then $p_{1} F$ is trivial, and hence the range of $F$ lies in $i_{1}(\pi(A))$, a discrete set. Connectedness of $U_{G} \times U_{A}$ and continuity of $F$ imply that $\alpha=\beta$.

The proof of (3) is trivial once (1) and (2) are established.

Thus the extension $D$ determines extensions $\widetilde{D}$ and $D_{\sharp}$. Now $\widetilde{D}$ and $D_{\ddagger}$ determine equivalence classes say

$$
[\widetilde{D}] \in H^{2}\left(U_{G}, U_{A}\right) \quad \text { and } \quad\left[D_{\sharp}\right] \in H^{2}(\pi(G), \pi(A)) .
$$

Proposition 1.4. Let $D=D(E, i, j)$ and $D^{\prime}=D^{\prime}\left(E^{\prime}, i^{\prime}, j^{\prime}\right)$ be two extensions of $G$ by $A$. Then if $D$ is equivalent to $D^{\prime},[\widetilde{D}]=\left[\widetilde{D}^{\prime}\right]$ and $\left[D_{\sharp}\right]=\left[D_{\sharp}^{\prime}\right]$.

Proof. Let $f$ be a topological isomorphism from $E$ onto $E^{\prime}$ such that $f i=i^{\prime}$ and $j^{\prime} f=j$. Then there exists a unique lifting $f$ of $\tilde{f}$ from $U_{E}$ onto $U_{E}$, such that $\tilde{f}$ is a topological isomorphism from $U_{E}$ onto $U_{E^{\prime}}, \widetilde{f} \tilde{i}=\widetilde{i}^{\prime}$, and $\tilde{j}^{\prime} \tilde{f}=\widetilde{j}$. Hence $\widetilde{f}$ establishes an equivalence between $\widetilde{D}$ and $\widetilde{D}^{\prime}$. Similarly on the homotopy level, $f_{\sharp}$ is an isomorphism between $\pi(E)$ and $\pi\left(E^{\prime}\right)$, and $f_{\sharp} i_{\sharp}=i_{\sharp}^{\prime}$ and $j_{\sharp}^{\prime} f_{\sharp}=j_{\sharp}$. Hence $\left[D_{\sharp}\right]=\left[D_{\sharp}^{\prime}\right]$.

Thus as a result of Proposition 1.4 we define mappings $\Phi_{1}$ and $\Phi_{2}$ each from $H^{2}(G, A)$ with $\Phi_{1}$ going into $H^{2}\left(U_{G}, U_{A}\right)$ where the action of $U_{G}$ on $U_{A}$ is the unique one compatible with the action of $G$ on $A$, and $\Phi_{2}$ going into $H^{2}(\pi(G), \pi(A))$ where the action of $\pi(G)$ on $\pi(A)$ is 
trivial, and where $\Phi_{1}([D])=[\widetilde{D}]$ and $\Phi_{2}([D])=\left[D_{\sharp}\right]$. Since $\pi(E)$ is always abelian, $\Phi_{2}$ actually maps $H^{2}(G, A)$ into the subgroup of $H^{2}(\pi(G), \pi(A))$ of the classes of symmetric extensions which we denote by $H_{s}^{2}(\pi(G), \pi(A))$. Combining $\Phi_{1}$ and $\Phi_{2}$ we obtain a mapping

$$
\Phi=\Phi_{1} \oplus \Phi_{2}: H^{2}(G, A) \longrightarrow H^{2}\left(U_{G}, U_{A}\right) \oplus H_{s}^{2}(\pi(G), \pi(A)) .
$$

In $\S 2$ we establish that $\Phi$ is a homomorphism. Before we do this we make an observation about the above construction. Note that we carefully choose the vertical extensions of Diagram 1 to be constructed in a specified manner (e.g., see [2]). This is important since there exist inequivalent extensions involving the universal covering group of a given group. For example, consider the following two inequivalent extensions of the 1 -torus, $T$, by the integers $Z$. Letting $R$ denote the real numbers we have:

$$
\begin{aligned}
& 1 \longrightarrow Z \stackrel{i}{\longrightarrow} R \stackrel{\exp }{\longrightarrow} T \longrightarrow 1 \text { and } \\
& 1 \longrightarrow Z \stackrel{-i}{\longrightarrow} R \stackrel{\exp }{\longrightarrow} T \longrightarrow 1 .
\end{aligned}
$$

Here exp is the exponential map of $R$ into $T, i$ is the identity injection of $Z$ into $R$, and $-i$ sends an integer $n$ into the real number $-n$. Extensions (1) and (2) are inequivalent. The first extension is the one obtained by following the procedures of [2]. One may ask whether the substitution of inequivalent vertical extensions in Diagram 1 will give rise to inequivalent horizontal extensions. There can be a certain ambiguity to the definition of $\Phi$ unless we specify how we are to choose the vertical extensions. We do have one result, however, which does simplify the problem and which will be useful in subsequent sections.

DEFINITION 1.5. Let $E$ be a connected, locally arcwise connected, and semi-locally simply connected group. Then the extension $V(F, p, q)$ is called a universal covering extension of $E$ if $F$ is a universal covering group for $E$ with projection $q$.

Proposition 1.6. Let $V_{2}^{\prime}(F, p, q)$ be any universal covering extension of the group $E$ in Diagram 1 . Let $K$ denote the domain of $p$. Then there exist unique continuous homomorphisms $r, s, h$, and $k$ such that $r: \pi(A) \rightarrow K s: K \rightarrow \pi(G), h: U_{A} \rightarrow F$, and $k: F \rightarrow U_{G}$, and such that the new diagram obtained from Diagram 1 is commutative. That is such that $p r=h i_{1}, q h=i p_{1}, i_{3} s=k p$, and $j q=p_{3} k$.

Furthermore, defining

$\mathrm{H}_{2}$ :

$$
1 \longrightarrow \pi(A) \stackrel{r}{\longrightarrow} K \stackrel{s}{\longrightarrow} \pi(G) \longrightarrow 1
$$


and $H_{1}: \quad \quad 1 \longrightarrow U_{A} \stackrel{h}{\longrightarrow} F \stackrel{k}{\longrightarrow} U_{G} \longrightarrow 1$

then $H_{1}$ and $H_{2}$ are extensions equivalent to $\widetilde{D}$ and $D_{\sharp}$ respectively (see Diagram 1).

Proof. Let us substitute $V_{2}^{\prime}$ for $V_{2}$ in the construction of Diagram 1. Then repeating earlier arguments we see that $i$ and $j$ induce homomorphisms $h: U_{A} \rightarrow F$ and $k: F \rightarrow U_{G}, \quad r: \pi(A) \rightarrow K$ and $s: K \rightarrow$ $\pi(G)$. Thus we obtain a new $3 \times 3$ commutative diagram which is the required one for this proposition. $H_{1}$ and $H_{2}$ are seen automatically to be extensions. The uniqueness of $h$ and $k$ follow from the type of topological argument involving connectedness and continuity which we have already seen. The proofs that $r$ and $s$ are unique involve simple algebraic arguments and are omitted. Hence we only have left to prove the equivalence of $H_{1}$ to $\widetilde{D}$ and $H_{2}$ to $D_{\sharp}$. Since $F$ and $U_{E}$ are simply connected there exists a unique topological isomorphism $\lambda: U_{B} \rightarrow F$ such that $p_{2}=q \lambda$. Since $p_{2} \tilde{i}=i p_{1}=q h$, we have that $q \lambda \tilde{i}=q h$. Hence for all $x \in U_{A}, q\left(\lambda \tilde{i}(x) h\left(x^{-1}\right)\right)=1$ and therefore $\lambda i(x) h\left(x^{-1}\right) \in p(K)$, a discrete set. Hence $\lambda \tilde{i}=h$. Similarly $\widetilde{j}=k \lambda$. Hence $H_{1}$ is equivalent to $\widetilde{D}$. Next observe that $\lambda i_{2}(\pi(E)) \subset$ $p(K)$. Hence define $\bar{\lambda}=p^{-1} \lambda i_{2}$. Then by a simple algebraic argument it is seen that $\bar{\lambda}$ is an isomorphism from $\pi(E)$ onto $K$ and that $\bar{\lambda} i_{ \pm}=r$ and $s \bar{\lambda}=j_{\ddagger}$. Hence $H_{2}$ is equivalent to $D_{\geqslant}$.

2. $\Phi$ is a homomorphism. The proof that $\Phi$ is a homomorphism necessitates the examination of an equivalent method of summing elements of $H^{2}(G, A)$ called the Baer sum. It involves the direct construction of a new group extension from two given extensions. Extension equivalence is a congruence with respect to this binary operation and hence there is defined a binary operation on $H^{2}(G, A)$, the Baer sum. This operation coincides with the group operation on $H^{2}(G, A)$. Their equivalence is shown in [8] for the nontopological case. For topological group extensions the same proof carries over, but with the appropriate measure theoretical arguments, applying the theory of Borel spaces. Since it is an integral part of our proof, we shall briefly outline the construction. Let $D_{1}\left(E_{1}, f_{1}, g_{1}\right)$ and $D_{2}\left(E_{2}, f_{2}, g_{2}\right)$ be two extensions of $G$ by $A$. Define

$$
\bar{E}=\left\{(x, y) \in E_{1} \times E_{2}: g_{1}(x)=g_{2}(y)\right\} .
$$

Define a map $\bar{j}: E \rightarrow G$ by $\bar{j}(x, y)=g_{1}(x)$. Then letting $\bar{i}$ denote $f_{1} \times f$ we obtain an extension 
of $G$ by $A \times A$, where $G$ acts on $A \times A$ pointwise. Next define $\Delta A \subset$ $A \times A$ by $\Delta A=\left\{\left(a, a^{-1}\right): a \in A\right\}$. Then $\Delta A$ is invariant under the action of $G$. Hence we may 'divide' the extension by $A$ obtaining a new extension

$$
1 \longrightarrow A \times A / \Delta A \stackrel{i^{\prime}}{\longrightarrow} \bar{E} / \bar{i}(\Delta A) \stackrel{j}{\longrightarrow} G \longrightarrow 1
$$

where $i^{\prime}$ and $j$ are the cannonical maps obtained from $\bar{i}$ and $\bar{j}$ as a result of taking quotients. Finally let $\theta_{A}: A \rightarrow A \times A / \Delta A$ be the isomorphism defined by taking the element $a$ into the coset $(a, 1) \triangle A$. Note that $\theta_{A}^{-1}((a, b) \Delta A)=a b$. Now define $i=i^{\prime} \theta_{A}$. Then we obtain an extension

$$
1 \longrightarrow A \stackrel{i}{\longrightarrow} \bar{E} / \bar{i}(\Delta A) \stackrel{j}{\longrightarrow} G \longrightarrow 1
$$

of $G$ by $A$. This extension is the required one whose equivalence class is the sum of $\left[D_{1}\right]$ and $\left[D_{2}\right]$. Naturally the same method of summing may be applied to compute sums for $H^{2}\left(U_{G}, U_{A}\right)$ and $H^{2}(\pi(G), \pi(A))$.

Our plan is to apply the method of construction simultaneously to the sum of two extensions of $G$ by $A$ and to the sum of each pair if images under $\Phi_{1}$ and $\Phi_{2}$. Consider the extensions $D_{1}$ and $D_{2}$ above. For each of these extensions we obtain a $3 \times 3$ commutative diagram as in Diagram 1. If we take the product of these two diagrams we obtain a new diagram, Diagram 2.

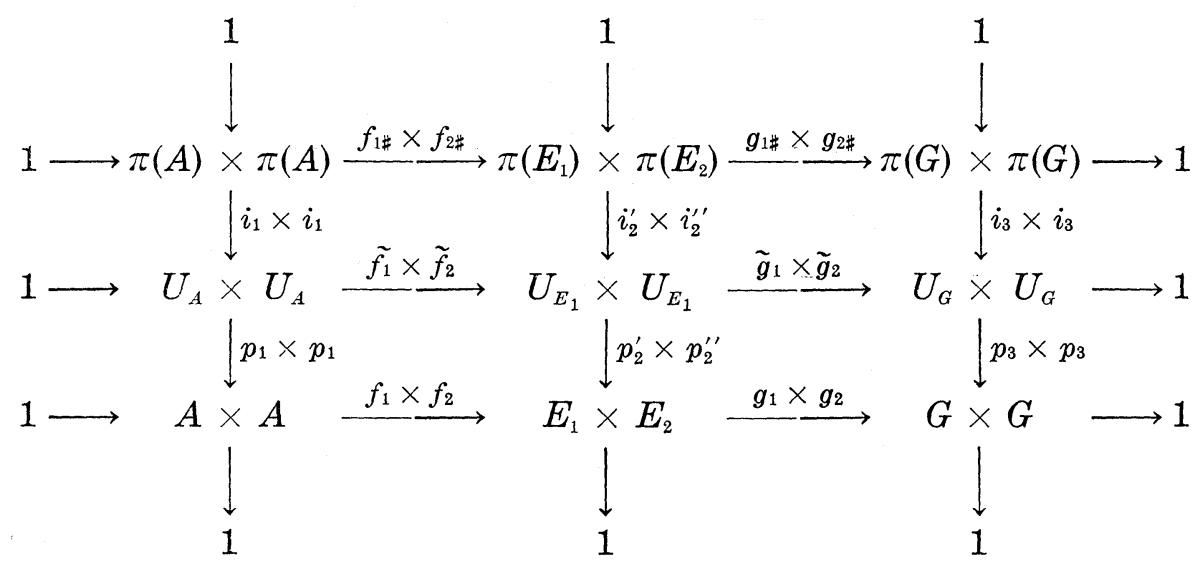

DiAgram 2.

If we now continue the process of simultaneously constructing the Baer sum in each of the three rows we obtain groups $\overline{\pi(E)}, \overline{U_{E}}$ and $\bar{E}$ defined by

$$
\begin{aligned}
\overline{\pi(E)} & =\left\{(a, b) \in \pi\left(E_{1}\right) \times \pi\left(E_{2}\right): g_{1 \sharp}(a)=g_{2 \sharp}(b)\right\}, \\
\overline{U_{E}} & =\left\{(x, y) \in U_{E_{1}} \times U_{E_{2}}: \widetilde{g}_{1}(x)=\widetilde{g}_{2}(y)\right\},
\end{aligned}
$$


and $\bar{E}$, defined as in the beginning of this section. Then also as in the beginning of this section we obtain maps, $\bar{i}, \widetilde{i}$, and $\bar{i}, \bar{j}, \widetilde{\vec{j}}$, and $\bar{j}_{\sharp}$. Then it is easily seen that, referring to Diagram 2 ,

$$
p_{2}^{\prime} \times p_{2}^{\prime \prime}\left(\overline{U_{E}}\right) \subset \bar{E}
$$

and $i_{2}^{\prime} \times i_{2}^{\prime \prime} \overline{(\pi(E))} \subset \bar{U}_{E}$. In fact it is easily seen that

$W:$

$$
1 \longrightarrow \overline{\pi(E)} \stackrel{i_{2}^{\prime} \times i_{2}^{\prime \prime}}{\longrightarrow} \overline{U_{E}} \stackrel{p_{2}^{\prime} \times p_{2}^{\prime \prime}}{\longrightarrow} \bar{E} \longrightarrow 1
$$

is an exact sequence and hence an extension. We thus obtain a commutative $3 \times 3$ diagram with the above sequence, $W$, as the middle vertical sequence, $V_{3}$ (see Diagram 1 ) as the right most vertical sequence, and the left vertical sequence coinciding with the left most vertical sequence of diagram 2. Next observe that $i_{1} \times i_{1}(\Delta \pi(A)) \subset \Delta U_{A}$ and $p_{1} \times p_{1}\left(\Delta U_{A}\right) \subset \Delta A$. Hence $i_{1} \times i_{1}$ induces a homomorphism $i_{1}^{\prime}$ from $\pi(A) \times \pi(A) / \Delta \pi(A)$ to $U_{A} \times U_{A} / \Delta U_{A}$. Also note that $i_{1}^{\prime} \theta_{\pi(A)}=\theta_{U_{A}} i_{1}$. Similarly we obtain a homomorphism $p_{1}^{\prime}$ such that $\theta_{A} p_{1}=p_{1}^{\prime} \theta_{U_{A}}$. Now if we devide the horizontal sequences by the appropriate $\Delta$ 's and substitute the extension $V_{1}$ of diagram 1 for the left most vertical extension we obtain a $3 \times 3$ diagram

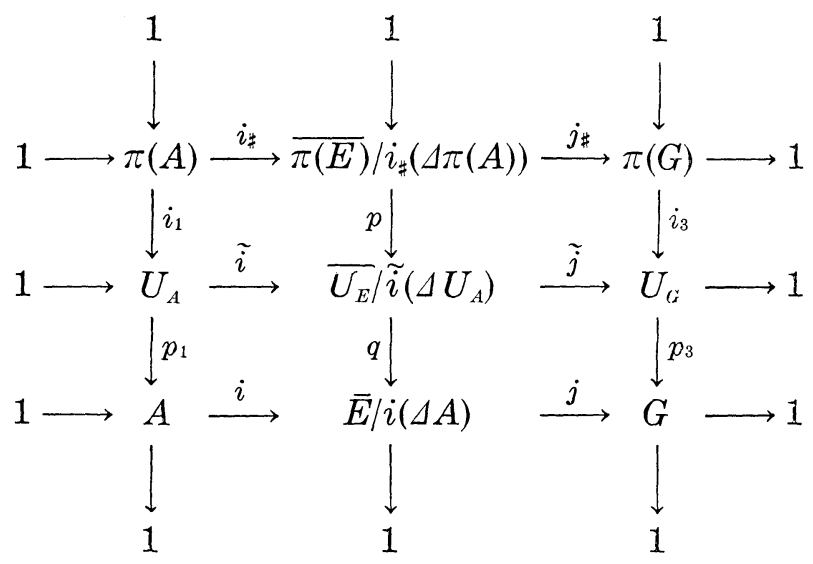

DIAGRAM 3.

where $p$ and $q$ are induced from $i_{1}^{\prime} \times i_{2}^{\prime}$ and $p_{1}^{\prime} \times p_{2}^{\prime}$ by taking quotients of $\overline{\pi(E)}$ by $i_{\geqslant}(\Delta \pi(A))$ and $\bar{U}_{E}$ by $\tilde{i}\left(\Delta U_{A}\right)$. It should be verified that the middle vertical sequence of diagram 3 is exact and that the entire diagram is commutative. We now need a few facts concerning topological properties of the middle vertical sequence.

Proposition 2.1. $\quad \bar{U}_{E} / \tilde{i}\left(\Delta U_{A}\right)$ is simply connected and $p$ embeds $\overline{\pi\left(E^{\prime}\right)} / i_{\#}(\Delta \pi(A))$ as a discrete subgroup. Hence the middle vertical sequence of Diagram 3 is a universal covering sequence for $\bar{E} / i(\Delta A)$. 
Proof. By a repetition of the argument of Proposition 1.1 it is easily seen that $\widetilde{j}: \bar{U}_{E} / \widetilde{i}\left(\Delta U_{A}\right) \rightarrow U_{G}$ is a fibre space with fibre $U_{A}$. Hence applying the homotopy sequence of a fibre space [5] and the fact that $U_{A}$ and $U_{G}$ are simply connected we obtain the first result. The second assertion follows from the fact that $\overline{\pi(E)} / i_{\sharp}(\Delta \pi(A))$ is itself discrete and $p$ embeds this discrete group homeomorphically into $\overline{U_{E}} / \tilde{i}\left(\Delta U_{A}\right)$. Hence the final result follows.

We are now prepared for the main result.

THEOREM 2.2. $\Phi_{1}, \Phi_{2}$ and hence $\Phi$ are homomorphisms.

Proof. Given the extensions $D_{1}$ and $D_{2}$ above, follow the construction outlined above to obtain Diagram 3. Then we obtain three horizontal extensions, the lowest belonging to the class $\left[D_{1}\right]+\left[D_{2}\right]$, the middle belonging to class $\Phi_{1}\left(\left[D_{1}\right]\right)+\Phi_{1}\left(\left[D_{2}\right]\right)$, and the top belonging to the class $\Phi_{2}\left(\left[D_{1}\right]\right)+\Phi_{2}\left(\left[D_{2}\right]\right)$. However by Propositions 1.6 and 2.1 the middle horizontal extension belongs to $\Phi_{1}\left(\left[D_{1}\right]+\left[D_{2}\right]\right)$ and the top horizontal extension belongs to class

$$
\Phi_{2}\left(\left[D_{1}\right]+\left[D_{2}\right]\right) .
$$

Hence $\Phi_{1}, \Phi_{2}$, and $\Phi$ are homomorphisms.

3. The range of $\Phi$. We have seen previously the range of $\Phi_{2}$ is contained in $H_{s}^{2}(\pi(G), \pi(A))$. We now attempt to find the range of $\Phi_{1}$. To begin with, observe that since $U_{G}$ acts on $U_{A}$ we may define an action of $\pi(G)$ on $U_{A}$ by mapping $(g, t)$ into $i_{3}(g) t$ for $g \in \pi(G)$ and $t \in U_{A}$.

Lemma 3.1. The action of $\pi(G)$ on $U_{A}$ defined above is trivial.

Proof. Let $D(E, i, j)$ be any extension of $G$ by $A$ (e.g., the trivial semidirect product). Then since the action of $U_{G}$ on $U_{A}$ is independent of the extension $D$ it suffices to show that $\pi(G)$ acts trivially on $U_{A}$ for this extension. Repeat the construction of $\S 1$ and obtain Diagram 1. Now let $a \in \pi(G)$. Choose a $b \in \pi(E)$ such that $j_{\sharp}(b)=a$. Then it is clear by commutativity that for $t \in U_{A}$,

$$
i_{3}(a) t=\tilde{i}^{-1}\left(i_{2}(b) \tilde{i}(t) i_{2}\left(b^{-1}\right)\right)=t
$$

since $i_{2}(\pi(E))$ lies in the center of $U_{E}$. Hence the result follows.

In the following lemmas we make use of the notation of Diagram 1. 
Lemma 3.2. Let $D$ be an extension of $G$ by $A$. Let $\alpha: \pi(G) \rightarrow \pi(E)$ be any cross section of $j_{\neq}$carrying the identity into the identity. Then there exists a Borel cross section $\beta: U_{F} \rightarrow U_{E}$ of $\tilde{j}$ such that $i_{2} \alpha=\beta i_{3}$.

Proof. First choose any Borel cross section $\beta^{\prime}$ of $\hat{j}$. Then define $\beta: U_{G} \rightarrow U_{E}$ by

(1) for $x \in i_{3}(\pi(G)) \quad \beta(x)=i_{2} \alpha i_{3}^{-1}(x)$

(2) otherwise define $\beta(x)=\beta^{\prime}(x)$.

Then it is clear that $\beta$ is Borel and satisfies the conditions of the lemma.

Definition 3.3. (1) Let $Z_{c}^{2}\left(i_{3}, U_{i}, U_{A}\right)$ denote the subgroup of $Z^{2}\left(U_{i}, U_{A}\right)$ consisting of all 2-cocycles $\sigma$ such that

$$
\sigma\left(i_{3}(g), s\right)=\sigma\left(x, i_{3}(g)\right)
$$

for all $g \in \pi(G)$ and $x \in U_{G}$.

(2) Let $H_{c}^{2}\left(i_{3}, U_{G}, U_{A}\right)$ be the subgroup of $H^{2}\left(U_{G}, U_{A}\right)$ obtained as the image of $Z_{c}^{2}\left(i_{3}, U_{G}, U_{A}\right)$ under the quotient homomorphism, mapping $Z^{2}\left(U_{G}, U_{A}\right)$ onto $Z^{2}\left(U_{G}, U_{A}\right) / B^{2}\left(U_{G}, U_{A}\right)=H^{2}\left(U_{G}, U_{A}\right)$.

Lemma 3.4. Let $D$ be an extension of $G$ by $A$. Let $\widetilde{D}$ be the resulting extension of $U_{G}$ by $U_{A}$ obtained in Diagram 1 . Then there exists a Borel cross section $\beta: U_{G} \rightarrow U_{L}$ of $\widetilde{j}$ such that the 2-cocycle obtained from $\beta$, $\nu$, defined by $\nu(x, y)=\widetilde{i}^{-1}\left(\beta(x) \beta(y) \beta(x y)^{-1}\right)$ lies in $Z_{c}^{2}\left(i_{3}, U_{i}, U_{A}\right)$.

Proof. Let $D_{\ddagger}$ be the resulting extension of $\pi(G)$ by $\pi(A)$ and let $\alpha$ be a cross section of $j_{\#}$ mapping the identity into the identity. Then by Lemma 3.2 there exists a Borel cross section $\beta: U_{G} \rightarrow U_{E}$ of $\widetilde{j}$ such that $i_{2} x=\beta i_{3}$. Then observe that since $i_{2}(\pi(E))$ lies in the center of $U_{L}$ that

$$
\beta i_{3}(g) \beta(x)=i_{2} \alpha(g) \beta(x)=\beta(x) i_{2} \alpha(g)=\beta(x) \beta i_{3}(g)
$$

for all $x \in U_{G}$ and $g \in \pi(G)$. Hence since $i_{3}(\pi(G))$ lies in the center of $U_{G}$ it is easily seen that $\nu\left(i_{3}(g), x\right)=\nu\left(x, i_{3}(g)\right)$ for all $x \in U_{G}$ and $g \in$ $\pi(G)$. This proves the result.

Corollary 3.5. $\Phi_{1}\left(H^{2}(G, A)\right) \subset H_{c}^{2}\left(i_{3}, U_{G}, U_{A}\right)$ and hence

$$
\Phi\left(H^{2}(G, A)\right) \subset H_{c}^{2}\left(i_{3}, U_{G}, U_{.}\right) \oplus H_{s}^{2}(\pi(G), \pi(A)) .
$$

We reserve the remainder of 83 for the argument asserting the converse of Corollary 3.5. We fix for the rest of the section extensions 
$H_{1}$ and $H_{2}$ such that $\left[H_{1}\right] \in H_{c}^{2}\left(i_{3}, U_{G}, U_{A}\right)$ and $\left[H_{2}\right] \in H_{s}^{2}(\pi(G), \pi(A))$. Suppose $H_{1}=H_{1}(F, h, k)$ and $H_{2}=H_{2}(K, r, s)$. Since

$$
\left[H_{1}\right] \in H_{c}^{2}\left(i_{3}, U_{G}, U_{A}\right)
$$

we can and do choose a Borel cross section $\beta$ of $k$ so that the cocycle $\nu$ determined by $\beta$ lies in $Z_{c}^{2}\left(i_{3}, U_{G}, U_{A}\right)$. Next we choose any cross section $\alpha$ of $s$ carrying the identity into the identity and let

$$
\mu \in Z_{s}^{2}(\pi(G), \pi(A))
$$

be the cocycle determined by $\alpha$. Then $r \mu(\alpha, b)=\alpha(\alpha) \alpha(b) \alpha(a b)^{-1}$ for $a$ and $b$ in $\pi(G)$ and $h \nu(x, y)=\beta(x) \beta(y) \beta(x y)^{-1}$ for $x$ and $y$ in $U_{G}$. We wish to put the extensions $H_{1}$ and $H_{2}$ together to construct an extension of $G$ by $A$. Our approach is to construct a homomorphism $p: K \rightarrow F$ which embeds $K$ into $F$ as a discrete central subgroup. By taking quotients we then obtain the required extension. The homomorphism $p$ is constructed in considerably more generality than is necessary. This is to allow for certain applications in the $\$ 4$.

Let Hom $\left(\pi(G), U_{A}\right)$ denote the set of all group homomorphisms from $\pi(G)$ into $U_{A}$.

Theorem 3.6. Let $\theta \in \operatorname{Hom}\left(\pi(G), U_{A}\right)$. Define $p: K \rightarrow F$ by

$$
p(x)=h\left(i_{1} r^{-1}\left(x(\alpha s(x))^{-1}\right) \theta s(x)\right) \beta i_{3} s(x) .
$$

Then

(1) $p r=h i_{1}, k p=i_{3} s$

(2) $p \alpha(a)=h \theta(a) \beta i_{3}(a)$ for $a \in \pi(G)$,

(3) $i_{1} \mu(a, b)=\nu\left(i_{3}(a), i_{3}(b)\right)$ for $a$ and $b$ in $\pi(G)$,

(4) $p$ is an injective homomorphism, and

(5) $p(K)$ is a central subgroup of $F$ if and only if the action of $U_{G}$ on $\theta(\pi(G))$ is trivial.

Proof. (1) and (2) follow by direct computation. A proof of (3) follows easily from (2) and (1) and the fact that each element of $\beta i_{3}\left(\pi(G)\right.$ ) commutes with each element of $h\left(U_{A}\right)$ (see Lemma 3.1). To prove (4) observe by computation (we omit the details), applying Lemma 3.1 when required, that

$$
p(x y)=h\left(i_{1} \mu(s(x), s(y)) \nu\left(i_{3} s(x), i_{3} s(y)\right)^{-1}\right) p(x) p(y) .
$$

That $p$ is a homomorphism then follows from the above equation and (3). To show that $p$ is injective let $x$ lie in the kernel of $p$. Then $k p(x)=i_{3} s(x)=1$. Hence since $i_{3}$ is injective $s(x)=1$. Hence for some $a \in \pi(A), r(a)=x$. Then $1=\operatorname{pr}(a)=h i_{1}(a)$. Since $h$ and $i_{1}$ are each injective the result follows. For the proof of (5) we first establish 
the following two lemmas.

LEMMA 1. $\beta i_{3}(\pi(G))$ lies in the center of $F$.

Proof. First let $x \in U_{G}$ and $a \in \pi(G)$. Then by hypothesis

$$
\nu\left(i_{3}(a), x\right)=\nu\left(x, i_{3}(a)\right) .
$$

Hence $\beta i_{3}(a) \beta(x)=\beta(x) \beta i_{3}(a)$. Now let $t \in F$. Then $t(\beta i(t))^{-1} \in h\left(U_{A}\right)$ and hence by Lemma 3.1 commutes with $\beta i_{3}(\alpha)$ for any $a \in \pi(G)$. Finally

$$
\begin{aligned}
\beta i_{3}(a) t & =\beta i_{3}(a)\left(t(\beta k(t))^{-1}\right) \beta k(t)=\left(t(\beta k(t))^{-1}\right) \beta i_{3}(a) \beta k(t) \\
& =\left(t(\beta k(t))^{-1}\right)(\beta k(t)) \beta i_{3}(a)=t \beta i_{3}(a)
\end{aligned}
$$

Lemma 2. The action of $U_{G}$ on $U_{A}$ is trivial on $i_{1}(\pi(A))$. Hence $h i_{1}(\pi(A))$ lies in the center of $F$.

Proof. First observe that $i_{1}(\pi(A))$ is invariant with respect to this action. For let $g \in U_{G}$ and $a \in i_{1}(\pi(A))$. Then

$$
p_{1}(g a)=p_{3}(g) p_{1}(a)=1 .
$$

Next fix any $a \in i_{1}(\pi(A))$. Then $g \rightarrow g a$ is a continuous function of connected set $U_{G}$ into discrete set $i_{1}(\pi(A))$. Since $a$ lies in the range of this function the result follows. The second statement then follows.

The proof of (5) now follows from Lemmas 1 and 2 and the fact that the action of $U_{G}$ on $\theta(\pi(G))$ is trivial if and only if $h \theta(\pi(G))$ lie in the center of $F$.

We are now prepared to put the extensions $H_{1}$ and $H_{2}$ together to obtain the required extension. In what follows we choose

$$
\theta \in \operatorname{Hom}\left(\pi(G), U_{A}\right)
$$

so that $U_{G}$ acts trivially on $\theta(\pi(G)$ ). (For example let $\theta$ be the trivial homomorphism.) Then by Theorem 3.6 we obtain an injective homomorphism $p$ of $K$ into the center of $F$ such that $p r=h i_{1}$, and $k p=$ $i_{3} s$. Let $q: F \rightarrow F / p(K)$ be the natural quotient homomorphism. As a quotient space $F / p(K)$ inherits both a topological and a Borel structure from $F$, and $q$ is both continuous and Borel. Now since

$$
h i_{1}(\pi(A)) \subset p(K) \text { and } k p(K) \subset i_{3}(\pi(G)),
$$

$p$ and $k$ induce unique homomorphisms $i$ and $j$ from $A$ to $F / p(K)$ and from $F / p(K)$ to $G$ respectively such that $i p_{1}=q h$ and $j q=p_{3} k$. It is also clear that $i$ and $j$ are continuous and Borel. Thus we obtain a sequence 
$D:$

$$
1 \longrightarrow A \stackrel{i}{\longrightarrow} F / p(K) \stackrel{j}{\longrightarrow} G \longrightarrow 1
$$

We are now prepared to formulate the main result of this section.

THEOREM 3.7. (1) $D$ is an extension of $G$ by $A$.

(2) $\Phi_{1}([D])=\left[H_{1}\right]$

(3) $\Phi_{2}([D])=\left[H_{2}\right]$.

Hence $\Phi$ maps $H^{2}(G, A)$ onto $H_{c}^{2}\left(i_{3}, U_{G}, U_{A}\right) \oplus H_{s}^{2}(\pi(G), \pi(A))$.

Proof. By the above we obtain a $3 \times 3$ commutative diagram whose top row is $H_{2}$, whose middle row is $H_{1}$, and whose bottom row is $D$. It is a simple matter, by chasing around this diagram, to verify that $D$ is exact. Since $i$ and $j$ are continuous to show that $D$ is an extension of $G$ by $A$ it suffices to show that $F / p(K)$ is a locally compact second countable Hausdorff space. This is true if and only if $p(K)$ is a closed subgroup of $F$. Thus it suffices to show that $F / p(K)$ is an analytic Borel space (see [1, p. 16]). (Note that the action of $U_{G}$ on $U_{A}$ will uniquely determine the action of $G$ on $A$ and hence we are guaranteed the correct action automatically). Since each of $A$ and $G$ are analytic Borel spaces it suffices to produce a bijective Borel map of $A \times G$ onto $F / p(K)$ whose inverse is also Borel. Let $\chi$ be a Borel cross section of $p_{3}$ carrying the identity into the identity. Define $\gamma: G \rightarrow F / q(K)$ by $\gamma=q \beta \chi$. Then it is easily verified that $\gamma$ is a Borel cross section of $j$ carrying the identity into the identity. Now define $\lambda: A \times G \rightarrow F / p(K)$ by $\lambda(a, g)=i(a) \gamma(g)$. Then $\lambda$ is clearly Borel. It is also clear that $\lambda$ is bijective and in fact

$$
\left.\lambda^{-1}(x)=\left(i^{-1}\left(x(\gamma j(x))^{-1}\right)^{-1}\right), j(x)\right) .
$$

Clearly $\lambda^{-1}$ is also Borel. Hence we conclude that $D$ is an extension of $G$ by $A$. Next note that since $F$ is simply connected and $K$ is discrete, the sequence

$$
1 \longrightarrow K \longrightarrow F \longrightarrow F / p(K) \longrightarrow 1
$$

is a universal extension of $F / p(K)$. Finally by the above, making use of Proposition 1.6 we conclude the theorem.

4. The kernel of $\Phi$. It is not always the case that $\Phi$ has trivial kernel. Let $S L_{2}(R)$ be the special linear group on $R^{2}$. Then it can be shown that $\Phi\left(H^{2}\left(S L_{2}(R), T\right)=0\right.$ where the action is trivial. In fact $H^{2}\left(S L_{2}(R), T\right) \cong T$. Our task is to compute the kernel of $\Phi$. in general. Consider any extension $D$ of $G$ by $A$ such that $\Phi([D])=0$. Now apply the techniques of $\S 1$ to $D$ to obtain Diagram I. Then the extensions $\widetilde{D}$ and $D_{\sharp}$ split. Hence there exist Borel cross sections. 
$\alpha$ and $\beta$ of $j_{\ddagger}$ and $\tilde{j}$ such that $\alpha$ and $\beta$ are each homomorphisms. Now for any $x \in \pi(G), i_{2} \alpha(x)\left(\beta i_{3}(x)\right)^{-1}$ lies in the kernel of $\tilde{j}$. Hence define $\theta(x)=i^{-1}\left(i_{2} \alpha(x)\left(\beta i_{3}(x)\right)^{-1}\right)$. It is clear that $\theta \in \operatorname{Hom}\left(\pi(G), U_{A}\right)$. Now by an argument similar to that used in Lemma 1 within the proof of Theorem 3.6 it is easily seen that $\beta i_{3}(\pi(G))$ lies in the center of $U_{E}$. Also $i_{2}(\pi(E))$ lies in the center of $U_{E}$. Hence the range of $\tilde{i} \theta$ lies in the center of $U_{E}$. Hence the action of $U_{G}$ on the range of $\theta$ is trivial.

DeFinition 4.1. Let Hom $\left(\pi(G), U_{A}{ }^{\prime} U_{G}\right.$ be the subgroup of Hom $\left(\pi(G), U_{A}\right)$ consisting of all elements $\theta$ such that the action of $U_{F}$ on $\theta(x)$ is trivial for each $x \in \pi(G)$.

DeFINITION 4.2. Let $\theta \in \operatorname{Hom}\left(\pi(G), U_{A}\right)_{U_{G}}$. Define $\rho(\theta) \in$ kernel $\Phi$ according to the following construction in the course of which we make use of the notation and results of Theorems 3.6 and 3.7. Let $H_{1}$ and $H_{2}$ be trivial extensions of $U_{G}$ by $U_{A}$ and $\pi(G)$ by $\pi(A)$. Let $\alpha$ and $\beta$ be splitting homomorphisms associated with $s$ and $k$. Then by Theorems 3.6 and $3.7 \theta$ determines an extension $D$ such that

$$
\Phi_{1}([D])=\left[H_{1}\right]=0 \text { and } \Phi_{2}([D])=\left[H_{2}\right]=0 \text {. }
$$

Thus $[D] \in$ kernel $\Phi$. Then define $\rho(\theta)=[D]$.

Proposition 4.3. (1) The definition of $\rho(\theta)$ is independent of the choice of splitting homomorphisms $\alpha$ and $\beta$. of $\Phi$.

(2) $\rho$ is a homomorphism of $\operatorname{Hom}\left(\pi(G), U_{A}\right)_{U_{G}}$ onto the kernel

Proof. Let $\chi: G \rightarrow U_{G}$ be a Borel cross section of $p_{3}$ carrying the identity into the identity. Let $\eta \in Z^{2}(G, \pi(G))$ be the cocycle associated with this cross section, i.e., $\eta(x, y)=i_{3}^{-1}\left(\chi(x) \chi(y) \chi(x y)^{-1}\right)$ for all $x$ and $y$ in $G$. We first establish the following lemma.

Lemma. Let $\sigma=p_{1} \theta \eta^{-1}$. Then $\sigma \in Z^{2}(G, A)$ and in fact $\sigma$ belongs to the cohomology class determined by $\rho(\theta)$.

Proof. We continue to use the notation of Theorems 3.6 and 3.7. Define $\gamma: G \rightarrow F / p(K)$ by $\gamma=q \beta \chi$. Then $\gamma$ is a Borel cross section of $j$ carrying the identity into the identity. Now define

$$
\sigma(x, y)=i^{-1}\left(\gamma(x) \gamma(y) \gamma(x y)^{-1}\right) .
$$

Then clearly $\sigma \in Z^{2}(G, A)$ and belongs to the cohomology class determined by $\rho(\theta)$. We shall show that $\sigma$ coincides with the expression stated in the lemma. For $x$ and $y$ in $G$, 


$$
\begin{aligned}
i \sigma(x, y) & =\gamma(x) \gamma(y) \gamma(x y)^{-1}=q \beta i_{3} \eta(x, y) \\
& =q\left(h \theta\left(\eta^{-1}(x, y)\right) p \alpha \eta(x, y)\right) \text { by Theorem 3.6-(2) } \\
& =q h \theta \eta^{-1}(x, y)=i p_{1} \theta \eta^{-1}(x, y)
\end{aligned}
$$

Hence $\sigma(x, y)=p_{1} \theta \eta^{-1}(x, y)$.

We now proceed with the proof of the proposition. Define a map. $\bar{\rho}:$ Hom $\left(\pi(G), U_{A}\right\rangle_{U G} \rightarrow Z^{2}(G, A)$ by $\bar{\rho}(\theta)=\sigma$ where $\sigma$ is as in the above lemma. Then by the result of the lemma it is easily seen that $\bar{\rho}$ is a homomorphism. Let $\left.\nu: Z^{2}, G, A\right) \rightarrow H^{2}(G, A)$ be the natural quotient map. Then the composition $\nu_{\bar{\rho}}$ is a homomorphism and is identical to $\rho$. Also by the equation of the lemma it is clear that $\rho$ is independent of $\alpha$ and $\beta$. Finally the proof that $\rho$ is onto follows easily from the introductory remarks of this section. For suppose [D] $\in$ kernel $\Phi$. Repeating the arguments at the beginning of this section we obtain trivial extensions $\widetilde{D}$ and $D_{\#}$ and splitting homomorphisms $\alpha$ and $\beta$, and $\theta \in \operatorname{Hom}\left(\pi(G), U_{A}\right)_{U_{G}}$ such that $\tilde{i} \theta(x) \beta i_{3}(x)=i_{2} \alpha(x)$ for all $x \in \pi(G)$. Next it is easily seen that if we construct the function $p: \pi(E) \rightarrow U_{E}$ using $\alpha, \beta$ and $\theta$ and the definition of $p$ given in Theorem 3.6 that $p=i_{2}$. Hence $\rho(\theta)=[D]$. This shows that $\rho$ is onto.

Hom $\left(\pi(G), U_{A}\right)_{U_{G}}$ is a vector group and hence is divisible. The homomorphic image of a divisable group is divisible and hence the kernel of $\Phi$ is divisible. Thus we obtain

COROLLARY 4.4. $H^{2}(G, A) \cong H_{c}^{2}\left(i_{3}, U_{G}, U_{A}\right) \oplus H_{s}^{2}(\pi(G), \pi(A)) \oplus$ kernel $\Phi$.

We now compute the kernel of $\rho$. Consider the extensions $V_{t}$ and $V_{3}$ in Diagram 1. Applying [10] we obtain a commutative diagram with rows and columns exact, Diagram 4.

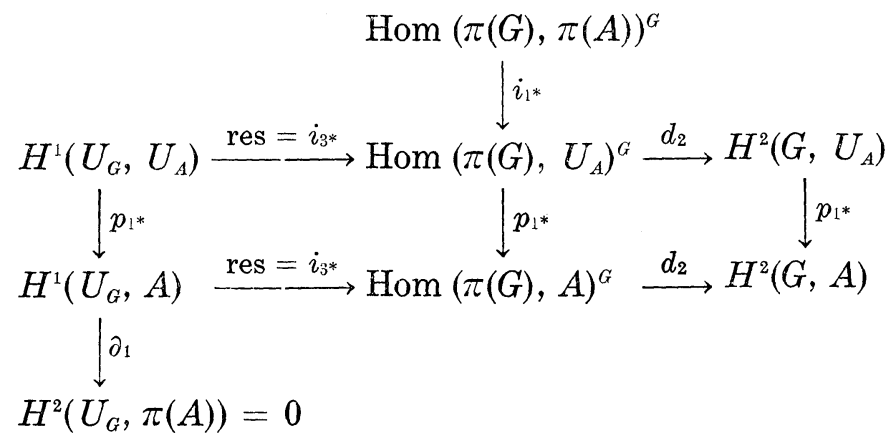

DIAGRAM 4.

Since $\pi(G)$ acts trivially on $\pi(A), U_{A}$, and $A$ the functor $H^{1}$ reduces to Hom in the appropriate column. Next observe that since $U_{G}$ is simply connected and $\pi(A)$ is discrete, $H^{2}\left(U_{G}, \pi(A)\right)=0$. Now 
let $\chi$ be as before, a Borel cross section of $p_{3}$ in extension $V_{3}$, such that $\chi$ carries the identity into the identity. Let $\eta \in Z^{2}(G, \pi(G))$ be the cocyle determined by $\chi$. Then as a 2-cocycle $\eta$ determines the extension $V_{3}$. Then by [10, p. 53] we have an explicit representation of $d_{2}: \operatorname{Hom}\left(\pi(G), U_{A}\right)^{G} \rightarrow H^{2}\left(G, U_{A}\right)$ in terms of cocycles. For

$$
\theta \in \operatorname{Hom}\left(\pi(G), U_{A}\right)^{G}
$$

and $x$ and $y$ in $G$, the cocycle taking $(x, y)$ into $\theta\left(\eta(x, y)^{-1}\right)$ belongs to the cohomology class $d_{2}(\theta)$. Consequently, by the lemma in the proof of Proposition 4.3 we have immediately (referring to Diagram 4)

\section{Proposition 4.5. $\rho=p_{1^{*}} d_{2}=d_{2} p_{1^{*}}$ on $\operatorname{Hom}\left(\pi(G), U_{A}\right)_{U_{G}}$.}

We now establish a few more preliminaries before obtaining our final result.

\section{Proposition 4.6. Referring to Diagram 4.}

$$
\operatorname{Hom}\left(\pi(G), U_{A}\right)^{G}=\operatorname{Hom}\left(\pi(G), U_{A}\right)_{U_{G}} .
$$

Proof. First note that since $\pi(G)$ acts trivially on $U_{A}$, the action of $U_{G}$ on $U_{A}$ determines an action of $G$ on $U_{A}$. For $\theta \in \operatorname{Hom}\left(\pi(G), U_{A}\right)$ and $g \in G g$ acts on $\theta$ in the following manner. For $a \in \pi(G)(g \theta)(a)=$ $g\left(\theta\left(g^{-1} a\right)\right)$. But $\pi(G)$ is central in $U_{G}$ and hence the action of $G$ on $\pi(G)$ is trivial. Hence $(g \theta)(a)=g(\theta(a))$. Hence $g \theta=\theta$ for all $g \in G$ if and only if $x$ acts trivially on the range of $\theta$ for all $x \in U_{G}$. The result follows.

TheOREM 4.7. The kernel of $\rho$ is

$$
i_{1^{*}}(\operatorname{Hom}(\pi(G), \pi(A))) \oplus i_{3}^{*}\left(H^{1}\left(U_{G}, U_{A}\right)\right) .
$$

Hence

$$
\operatorname{kernel} \Phi \cong \frac{\operatorname{Hom}\left(\pi(G), U_{A}\right)^{G}}{i_{1^{*}}(\operatorname{Hom}(\pi(G), \pi(A))) \oplus i_{3}^{*}\left(H^{1}\left(U_{G}, U_{A}\right)\right)}
$$

Proof. First note that since $U_{G}$ acts trivially on $\pi(A)$ so does $G$. Hence since $G$ acts trivially on $\pi(G)$,

$$
\text { Hom }(\pi(G), \pi(A))^{G}=\operatorname{Hom}(\pi(G), \pi(A)) .
$$

Hence by exactness and commutativity of Diagram 4 and by Lemma 4.6 it is clear that the kernel of $\rho$ contains the sum. Now let $a \in$ kernel $\rho$. Then by Proposition $4.5 d_{2} p_{1^{*}}(a)=0$. Then by a suitable argument involving chasing around Diagram 4 it is a simple matter 
to show that $a$ lies in this sum. This completes the proof.

5. Special cases. We now proceed to compute $H^{2}(G, A)$ in the light of the above structure theory under certain circumstances.

Proposition 5.1. If $G$ is compact or if $\pi(G)$ is a torsion group the kernel of $\Phi$ is 0 .

Proof. If $G$ is compact $H^{2}\left(G, U_{A}\right)=0$, since $U_{A}$ is a vector group (see Moore [10]). Hence by Proposition 4.5, $\rho$ is trivial. Since $\rho$ is surjective onto the kernel of $\Phi$ the result is assured.

If $\pi(G)$ is torsion then $\operatorname{Hom}\left(\pi(G), U_{A}\right)=0$, since $U_{A}$ is a vector group. Hence again by Proposition $4.5 \rho$ is trivial and the result follows.

The following generalizes Theorem A of [10].

Proposition 5.2. If $G$ is simply connected then

$$
p_{1^{*}}: H^{2}\left(G, U_{A}\right) \longrightarrow H^{2}(G, A)
$$

is an isomorphism.

Proof. By Proposition 5.1 the kernel of $\Phi$ is 0 . Clearly

$$
H_{s}^{2}(\pi(G), \pi(A))=0 .
$$

Since $i_{3}$ is trivial, $H_{c}^{2}\left(i_{3}, U_{G}, U_{A}\right)=H^{2}\left(U_{G}, U_{A}\right)$. Hence $\Phi$ is an isomorphism from $H^{2}(G, A)$ onto $H^{2}\left(U_{G}, U_{A}\right)$. Now since $G$ is simply connected $p_{3}$ is an isomorphism, and hence $p_{3}^{*}: H^{2}\left(G, U_{A}\right) \rightarrow H^{2}\left(U_{G}, U_{A}\right)$ is an isomorphism. Hence the composite $p_{3}^{*-1} \Phi$ is an isomorphism. We show that the inverse of this isomorphism is $p_{1^{*}}$. Let $D(E, i, j)$ be any extension of $G$ by $A$. Then $\Phi([D])=[\widetilde{D}]$ (using the usual notation of Diagram 1). Let $\beta: U_{G} \rightarrow U_{E}$ be a Borel cross section of $\tilde{j}$ carrying the identity into the identity. Then define $\gamma: G \rightarrow E$ by $\gamma=$ $p_{2} \beta p_{3}^{-1}$. Then $\gamma$ is a Borel cross section of $j$ carrying the identity into the identity. Define $\sigma$ and $\nu$ by the equations

$$
\begin{aligned}
& \sigma(x, y)=i^{-1}\left(\gamma(x) \gamma(y) \gamma(x y)^{-1}\right) \\
& \nu(x, y)=i^{-1}\left(\beta(x) \beta(y) \beta(x y)^{-1}\right) .
\end{aligned}
$$

Then $\sigma$ and $\nu$ are the cocycles which determine the extensions $D$ and $\widetilde{D}$ respectively. It is easily verified that $\sigma\left(p_{3}(x), p_{3}(y)\right)=p_{1} \nu(x, y)$ for all $x$ and $y$ in $U_{G}$. Hence on the cohomology level, $p_{3}^{*}([\sigma])=p_{1^{+}}([\nu])$. But $[\nu]=\Phi([\sigma])$. Hence $p_{1^{*}} \Phi=p_{3}^{*}$. Hence we obtain Diagram 5, with lower right part commutative, as is the entire diagram excluding $\Phi$. 


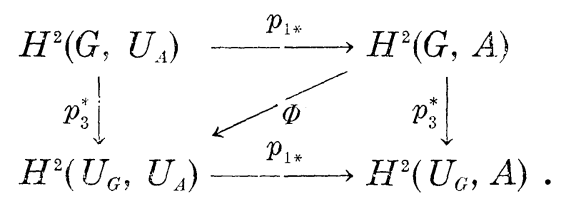

DIAGRAM 5.

Now let $\lambda \in H^{2}\left(G, U_{A}\right)$. Then $p_{1^{*}} \Phi p_{1^{*}}(\lambda)=p_{3}^{*} p_{1^{*}}(\lambda)=p_{1^{*}} p_{3}^{*}(\lambda)$. Hence $\Phi p_{1^{*}}(\lambda)-p_{3}^{*}(\lambda)$ lies in the kernel of $p_{1^{*}}: H^{2}\left(U_{G}, U_{A}\right) \rightarrow H^{2}\left(U_{G}, A\right)$. Since $U_{G}$ is simply connected and $\pi(A)$ is discrete, $H^{2}\left(U_{G}, \pi(A)\right)=0$ and hence by [10] $p_{1^{*}}$ has trivial kernel. Hence the upper left-hand part of Diagram 5 is commutative and result follows.

Proposition 5.3. If $G$ is compact $H_{c}^{2}\left(i_{3}, U_{G}, U_{A}\right)=0$ and hence $\left.\tilde{\Phi}: H^{2}, G, A\right) \cong H_{s}^{2}(\pi(G), \pi(A))$.

Proof. Since $G$ is compact and $H^{1}\left(\pi(G), U_{A}\right)$ is a vector group, $H^{1}\left(G, H^{1}\left(\pi(G), U_{A}\right)\right)=0$ by [10]. Hence again by [10, p. 47] the sequence

$$
H^{2}\left(G, U_{A}\right) \stackrel{p_{3^{*}}}{\longrightarrow} H^{2}\left(U_{G}, U_{A}\right) \stackrel{i_{3^{*}}}{\longrightarrow} H^{2}\left(\pi(G), U_{A}\right)^{G}
$$

is exact. Again by [10] since $G$ is compact $H^{2}\left(G, U_{A}\right)=0$. Hence $i_{3}^{*}$ is injective. In particular the subgroup $H_{c}^{2}\left(i_{3}, U_{G}, U_{A}\right)$ injects by $i_{3}^{*}$ into $H^{2}\left(\pi(G), U_{A}\right)$. However observe that the image under $i_{3}^{*}$ of an element of $H_{c}^{2}\left(i_{3}, U_{G}, U_{A}\right)$ actually lies in $H_{s}^{2}\left(\pi(G), U_{A}\right)$, the family of classes of symmetric extensions of $\pi(G)$ by $U_{A}$. This is so since the action of $\pi(G)$ on $U_{A}$ is trivial. But $U_{A}$ is divisable and hence any abelian extension of $\pi(G)$ by $U_{A}$ splits. Since $\pi(G)$ is discrete any such extension will automatically be topological. Hence $H_{s}^{2}\left(\pi(G), U_{A}\right)=0$. We conclude that $H_{c}^{2}\left(i_{3}, U_{G}, U_{A}\right)=0$. The result now follows from Proposition 5.1.

We now further investigate the relationship between $\left.H_{c}{ }^{\prime} i_{3}, U_{(i}, U_{A}\right)$ and $H^{2}\left(U_{G}, U_{A}\right)$.

Definition 5.4. Fix $\nu \in Z^{2}\left\{U_{G}, U_{A}\right\}$. For $a \in \pi(G)$ and $x \in U_{G}$ define $L(\nu)_{a}(x)=\nu\left(i_{3}(a), x\right) \nu\left(x, i_{3}(a)\right)^{-1}$.

THEOREM 5.5. (1) For each $a \in \pi(G)$ and $\nu \in Z^{2}\left(U_{G}, U_{A}\right), L(\nu)_{a} \in$ $Z^{1}\left(U_{G}, U_{A}\right)$.

(2) Define $\bar{L}(\nu)_{a}$ to be the cohomology class to which $L(\nu)_{a}$ belongs. Then $\bar{L}(\nu)$ is a homomorphism of $\pi(G)$ into $H^{1}\left(U_{G}, U_{A}\right)$.

(3) $\bar{L}$ is a homomorphism from $Z^{2}\left(U_{G}, U_{A}\right)$ into

$$
\operatorname{Hom}\left(\pi(G), H^{1}\left(U_{G}, U_{A}\right)\right) \text {, }
$$


and $B^{2}\left(U_{G}, U_{A}\right)$ lies in the kernel of $\bar{L}$.

(4) Hence $\bar{L}$ determines a homomorphism $T$ of $H^{2}\left(U_{G}, U_{A}\right)$ into $\operatorname{Hom}\left(\pi(G), H^{1}\left(U_{G}, U_{A}\right)\right)$.

(5) The kernel of $T$ is precisely $H_{c}^{2}\left(i_{3}, U_{G}, U_{A}\right)$.

Proof. We omit most of the computational details necessary to establish the theorem. The reader is referred to the identity (2) of p. 171 of G. W. Mackey [7] and the fact that $\pi(G)$ acts trivially on $U_{A}$. From these facts the following identities can easily be derived: For $\nu \in Z^{2}\left(U_{G}, U_{A}\right), a \in \pi(G)$ and $x$ and $y$ in $U_{G}$ :

(1) $L(\nu)_{a}(x y)=\left(L(\nu)_{a}(x)\right)\left(x L(\nu)_{a}(y)\right)$

(2) $\mathrm{L}(\nu)_{a b}(x)=L(\nu)_{a}(x) L_{(}^{\prime}(\nu)_{b}(x)\left(x \nu\left(i_{3}(a), i_{3}(b)\right)\right) \nu\left(i_{3}(a), i_{3}(b)\right)^{-1}$.

Part 1 then follows immediately from equation (1) above. Since the mapping taking $x \in U_{G}$ into $x \nu\left(i_{3}(a), i_{3}(b)\right) \nu\left(i_{3}(a), i_{3}(b)\right)^{-1}$ belongs to $B^{1}\left(U_{G}, U_{A}\right)$ it easily follows from identity (2) above that $\bar{L}(\nu)$ is a homomorphism. This proves 2 . It is a trivial matter that $\bar{L}$ is a homomorphism. Next let $\theta$ be any Borel mapping from $U_{G}$ to $U_{A}$ carrying the identity into the identity. Then $\theta$ determines a coboundary $\eta_{0}$ satisfying equation (3) of [7, p. 172]. Then for $a \in \pi(G)$ and $x \in U_{G}$ it is easily seen that $L\left(\eta_{0}\right)_{a}(x)=\theta\left(i_{3}(a)\right)\left(x \theta\left(i_{3}(a)\right)\right)^{-1}$ and hence $L\left(\eta_{0}\right)_{a}$ lies in $B^{1}\left(U_{G}, U_{A}\right)$. Hence $\bar{L}\left(\eta_{0}\right)=0$. This proves 3 . The proof of 4 is now immediate, $T$ being the obvious induced mapping on the quotient.

We now establish 5. Let $\lambda \in H_{c}^{2}\left(i_{3}, U_{G}, U_{A}\right)$. Then there exists an element $\nu \in Z_{c}^{2}\left(i_{3}, U_{G}, U_{A}\right)$ such that $\nu \in \lambda$. Hence

$$
\nu\left(i_{3}(a), x\right) \nu^{\prime}\left(x, i_{3}(a)\right)^{-1}=1
$$

for all $a \in \pi(G)$ and $x \in U_{G}$. Hence $T(\lambda)=0$. Conversely suppose $\lambda \epsilon$ kernel $T$. Choose a cocycle $\nu$ in $\lambda$. Then $L(\nu): \pi(G) \rightarrow B^{1}\left(U_{G}, U_{A}\right)$. Hence for each $a \in \pi(G)$ there exists a $t_{a} \in U_{A}$ such that

$$
L(\nu)_{a}(x)=\left(x t_{a}\right) t_{a}^{-1}
$$

for all $x \in U_{G}$. Thus $a \rightarrow t_{a}$ determines a map from $\pi(G)$ into $U_{G}$, and it can be taken so that $t_{1}=1$. This map can then be 'extended' to a Borel function $\phi$ from $U_{G}$ into $U_{A}$ satisfying the condition that $\phi\left(i_{3}(a)\right)=t_{a}$ for all $a \in \pi(G)$. Then $\dot{\phi}$ determines a coboundary $\delta \dot{\phi} \epsilon$ $B^{2}\left(U_{G}, U_{A}\right)$. Let $\nu^{\prime}=\nu+\delta \phi \in Z^{2}\left(U_{G}, U_{A}\right)$. Then

$$
L\left(\nu^{\prime}\right)_{a}(x)=L(\nu)_{a}(x) \phi\left(i_{3}(a)\right)\left(x \phi\left(i_{3}(a)\right)\right)^{-1}=x t_{a}\left(t_{a}\right)^{-1} t_{a}\left(x t_{a}\right)^{-1}=1 .
$$

Hence $\nu^{\prime}\left(i_{3}(a), x\right)=\nu_{(}^{\prime}\left(x, i_{3}(a)\right)$. Hence $\nu^{\prime} \in Z_{c}^{2}\left(i_{3}, U_{G}, U_{A}\right)$. Hence

$$
\left[\nu^{\prime}\right]=[\nu]=\lambda \in H_{c}^{2}\left(i_{3}, U_{G}, U_{A}\right) \text {. }
$$

Corollary 5.6. If $\pi(G)$ is a torsion group then $H_{c}^{2}\left(i_{3}, U_{G}, U_{A}\right)=$ 
$H^{2}\left(U_{G}, U_{A}\right)$.

DEFINITION 5.7. An abelian group $H$ is almost torsion if the quotient group $H /$ torsion $H$ is cyclic.

LEMMA 5.8. If $\pi(G)$ is almost torsion then $L(\nu)_{a}\left(i_{3}(b)\right)=1$ for all $\nu \in Z^{2}\left(U_{G}, U_{A}\right)$ and $a$ and $b$ in $\pi(G)$.

Proof. Fix $a \nu \in Z^{2}\left(U_{G}, U_{A}\right)$ and $a$ and $b$ in $\pi(G)$. Then there exist elements $r, s$, and $t$ in $\pi(G)$ such that $s$ and $t$ are torsion elements and there exist integers $m$ and $n$ such that $a=r^{m} s$ and $b=$ $r^{n} t$. Then $\bar{L}(\nu)_{a}=\bar{L}(\nu)_{r m_{s}}=m \bar{L}(\nu)_{r}+\bar{L}(\nu)_{s}=m \bar{L}(\nu)_{r}$ since $s$ is torsion and $H^{1}\left(U_{G}, U_{A}\right)$ is torsion free.

Now $i_{3}$ induces a map on the cohomology level $i_{3}^{*}: H^{1}\left(U_{G}, U_{A}\right) \rightarrow$ $H^{1}\left(\pi(G), U_{A}\right)$. Now since $\pi(G)$ acts trivially on $U_{A}, B^{1}\left(\pi(G), U_{A}\right)=0$ and $Z^{1}\left(\pi(G), U_{A}\right)$ is Hom $\left(\pi(G), U_{A}\right)$, the group of homomorphisms. Consequently the composition $L(\nu)_{r} i_{3}$ is a homomorphism. Hence

$$
\begin{aligned}
L(\nu)_{a}\left(i_{3}(b)\right) & =\left(m L(\nu)_{r}\right)\left(i_{3}(b)\right)=\left(m L(\nu)_{r}\right)\left(i_{3}\left(r^{n} t\right)\right) \\
& =\left(m L(\nu)_{r}\right)\left(i_{3}\left(r^{n}\right)\right)=\left(m n L(\nu)_{r}\right)\left(i_{3}(r)\right) .
\end{aligned}
$$

But $\left(m n L(\nu)_{r}\right) i_{3}(r)=\left(\nu\left(i_{3}(r), i_{3}(r)\right) \nu\left(i_{3}(r), i_{3}(r)\right)^{-1}\right)^{m n}=1$. Hence the result.

THEOREM 5.9. If $\pi(G)$ is almost torsion and $G$ is compact $T=$ 0 and hence $H_{c}^{2}\left(i_{3}, U_{G}, U_{A}\right)=H^{2}\left(U_{G}, U_{A}\right)=0$.

Proof. Fix $\nu \in Z^{2}\left(U_{G}, U_{A}\right)$. Then by Lemma $5.8, i_{3}^{*} \bar{L}(\nu)$ is trivial. Now by [10] the sequence

$$
0 \longrightarrow H^{1}\left(G, U_{A}\right) \stackrel{p_{3^{*}}}{\longrightarrow} H^{1}\left(U_{G}, U_{A}\right) \stackrel{i_{3^{*}}}{\longrightarrow} H^{1}\left(\pi(G), U_{A}\right)^{G}
$$

is exact and hence $\bar{L}(\nu)$ lies in the image of $p_{3}^{*}$. But since $G$ is compact and $U_{A}$ is a vector group $H^{1}\left(G, U_{A}\right)=0$ by [10]. Hence $\bar{L}(\nu)$ is trivial. We conclude that $T$ is trivial and hence the result follows.

The following theorem which we state for central extensions does not seem to generalize for arbitrary topological extensions.

THEOREM 5.10. Suppose the action of $G$ on $A$ is trivial. Then if $G$ is semisimple

$$
H_{c}^{2}\left(i_{3}, U_{G}, U_{A}\right)=H^{2}\left(U_{G}, U_{A}\right) .
$$


Proof. Let $\nu \in Z^{2}\left(U_{G}, U_{A}\right)$. Then since the action of $U_{G}$ on $U_{A}$ is trivial $L(\nu)_{a}$ is a homomorphism of $U_{G}$ into $U_{A}$ for each $a \in \pi(G)$. Now since $G$ is semisimple so is $U_{G}$. Hence $L(\nu)_{a}$ is trivial for each $\nu \in Z^{2}\left(U_{G}, U_{A}\right)$ and for each $a \in \pi(G)$. Hence $T$ is trivial and the result follows.

Recall by Proposition 5.3 that if $G$ is compact

$$
\Phi: H^{2}(G, A) \cong H_{s}^{2}(\pi(G), \pi(A)) \text {. }
$$

Now $\pi(A)$ is a finite product of copies of the integers, $Z$ and hence $H_{s}^{2}(\pi(G), \pi(A))$ is a finite product of copies of $H_{s}^{2}(\pi(G), Z)$.

Proposition 5.11. If $\pi(G)$ is finitely generated then

$$
H^{2}(\pi(G), Z)=H_{s}^{2}(\pi(G), Z) \cong(\operatorname{torsion} \pi(G))^{\wedge},
$$

the Pontryagin dual of the torsion subgroup of $\pi(G)$.

Proof. Since $\pi(G)$ is finitely generated $\pi(G) \cong F \oplus K$ where $F$ is free and $K$ is the torsion part of $\pi(G)$. Then by [8] $H^{2}(F, Z)=0$ and $H^{2}(K, Z) \cong \hat{K}$. From the exactness of the sequence,

$$
1 \longrightarrow F \stackrel{i}{\longrightarrow} \pi(G) \stackrel{j}{\longrightarrow} K \longrightarrow 1
$$

we obtain, assuming that all group actions are trivial,

$$
\begin{aligned}
& \longrightarrow \operatorname{Hom}(\pi(G), Z) \stackrel{i^{*}}{\longrightarrow} \operatorname{Hom}(F, Z) \stackrel{d_{2}}{\longrightarrow} H^{2}(K, Z) \\
& \stackrel{j^{*}}{\longrightarrow} H^{2}(\pi(G), Z) \longrightarrow 0 \text {. }
\end{aligned}
$$

Next note that since $K$ is torsion $i^{*}$ is an isomorphism. Thus we obtain the exactness of the sequence

$$
0 \stackrel{d_{2}}{\longrightarrow} H^{2}(K, Z) \stackrel{j^{*}}{\longrightarrow} H^{2}(\pi(G), Z) \longrightarrow 0 .
$$

Hence $j^{*}$ is an isomorphism and we conclude that $H^{2}(\pi(\pi(G), Z) \cong \hat{K}$. Next observe that $H^{2}(K, Z)=H_{s}^{2}(K, Z)$. For let

$$
1 \longrightarrow Z \stackrel{i}{\longrightarrow} B \stackrel{j}{\longrightarrow} K \longrightarrow 1
$$

be any central extension $K$ by $Z$. Let $\sigma: K \times K \rightarrow Z$ be any cocycle associated with this extension. Fix $a \in K$. Then define

$$
L_{a}(x)=\sigma(a, x) \sigma(x, a)^{-1}
$$

for $x \in K$. Then $L_{a}: K \rightarrow Z$ is a homomorphism. Hence since $K$ is torsion and $Z$ is free, $L_{a}$ is trivial for all $a \in K$. Hence $\sigma$ is symmetric and therefore $H^{2}(K, Z)=H_{s}^{2}(K, Z)$. 
Finally $j^{*}$ maps symmetric cohomology classes onto symmetric classes and hence since $j^{*}$ is an isomorphism,

$$
H^{2}(\pi(G), Z)=H_{s}^{2}(\pi(G), Z) .
$$

The author wishes to express his appreciation to Prof. C. C. Moore under whose direction this research was done.

\section{BIBLIOGRAPHY}

1. L. Auslander and C. C. Moore, Unitary representations of solvable Lie groups, Memoirs Amer. Nath. Soc. 62 Providence, 1966.

2. C. Chevalley Lie Groups, Princeton University Press, 1946.

3. A. Gleason, Spaces with a compact Lie group of transformations, Proc. Amer. Math. Soc. 1 (1950), 35-43.

4. G. Hochschild, Group extensions of Lie groups, Ann. of Math. (2) 54 (1951), 96109.

5. S. Hu, Homotopy Theory, Academic Press, New York, 1959.

6. G. W. Mackey, Borel structures in groups and their duals, Trans. Amer. Math. Soc. 85 (1957), 134-165.

7. _ Les ensembles Boreliens et les extensions des groupes, J. Math. Pures Appl. 36 (1957), 171-178.

8. S. MacLane, Homology Theory, Springer-Verlag, New York, 1967.

9. D. Montgomery and L. Zippen, Topological Transformation Groups, Interscience, New York, 1955.

10. C. C. Moore, Extensions and low dimensional cohomology theory of locally compact groups, I, Trans. Amer. Math. Soc. (1) 113 (1964), 40-63.

11. J. P. Serre, Trivialite des espaces fibres. Applicaiions, Comptes Rendus de L'Academie des Sciences Paris (10) 230 (1950), 916-918.

Received January 12, 1970.

ILLINOIS STATE UNIVERSITY 


\section{PACIFIC JOURNAL OF MATHEMATICS}

\section{EDITORS}

H. SAMELson

Stanford University

Stanford, California 94305

R. R. PHelPS

University of Washington

Seattle, Washington 98105
J. Dugundu

Department of Mathematics

University of Southern Californle

Los Angeles, California 9.0007

RICHARD ARENS

University of California

Los Angeles, California 9.0024

\section{ASSOCIATE EDITORS}
E. F. BECKENBACH
B. H. NeumanN
F. WOLE
K. Yoshida

\section{SUPPORTING INSTITUTIONS}

UNIVERSITY OF BRITISH COLUMBIA

CALIFORNIA INSTITUTE OF TECHNOLOGY

UNIVERSITY OF CALIFORNIA

MONTANA STATE UNIVERSITY

UNIVERSITY OF NEVADA

NEW MEXICO STATE UNIVERSITY

OREGON STATE UNIVERSITY

UNIVERSITY OF OREGON

OSAKA UNIVERSITY

UNIVERSITY OF SOUTHERN CALIFORNIA

\author{
STANFORD UNIVERSITY \\ UNIVERSITY OF TOKYO \\ UNIVERSITY OF UTAH \\ WASHINGTON STATE UNIVERSITY \\ UNIVERSITY OF WASHINGTON \\ AMERICAN MATHEMATICAL SOCIETY \\ CHEVRON RESEARCH CORPORATION \\ NAVAL WEAPONS CENTER
}

The Supporting Institutions listed above contribute to the cost of publication of this Journal, but they are not owners or publishers and have no responsibility for its content or policies.

Mathematical papers intended for publication in the Pacific Journal of Mathematics should be in typed form or offset-reproduced, (not dittoed), double spaced with large margins. Underline Greek letters in red, German in green, and script in blue. The first paragraph or two must be capable of being used separately as a synopsis of the entire paper. The editorial "we" must not be used in the synopsis, and items of the bibliography should not be cited there unless absolutely necessary, in which case they must be identified by author and Journal, rather than by item number. Manuscripts, in duplicate if possible, may be sent to any one of the four editors. Please classify according to the scheme of Math. Rev. Index to Vol. 39. All other communications to the editors should be addressed to the managing editor, Richard Arens, University of California, Los Angeles, California, 90024.

50 reprints are provided free for each article; additional copies may be obtained at cost in multiples of 50 .

The Pacific Journal of Mathematics is published monthly. Effective with Volume 16 the price per volume (3 numbers) is $\$ 8.00$; single issues, $\$ 3.00$. Special price for current issues to individual faculty members of supporting institutions and to individual members of the American Mathematical Society: $\$ 4.00$ per volume; single issues $\$ 1.50$. Back numbers are available.

Subscriptions, orders for back numbers, and changes of address should be sent to Pacific Journal of Mathematics, 103 Highland Boulevard, Berkeley, California, 94708.

PUBLISHED BY PACIFIC JOURNAL OF MATHEMATICS, A NON-PROFIT CORPORATION

Printed at Kokusai Bunken Insatsusha (International Academic Printing Co., Ltd.), 7-17, Fujimi 2-chome, Chiyoda-ku, Tokyo, Japan. 


\section{Pacific Journal of Mathematics}

\section{Vol. 36, No. 2 December, 1971}

George E. Andrews, On a partition problem of H. L. Alder ............ 279

Thomas Craig Brown, An interesting combinatorial method in the theory of locally finite semigroups .......................... 285

Yuen-Kwok Chan, A constructive proof of Sard's theorem ............. 291

Charles Vernon Coffman, Spectral theory of monotone Hammerstein

operators...................................... 303

Edward Dewey Davis, Regular sequences and minimal bases .......... 323

Israel (Yitzchak) Nathan Herstein and Lance W. Small, Regular elements in

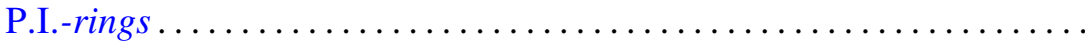

Marcel Herzog, Intersections of nilpotent Hall subgroups ..............

W. N. Hudson, Volterra transformations of the Wiener measure on the space

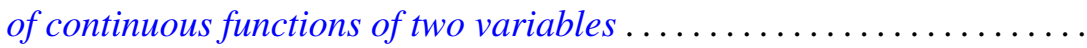

J. H. V. Hunt, An n-arc theorem for Peano spaces ................ 351

Arnold Joseph Insel, A decomposition theorem for topological group

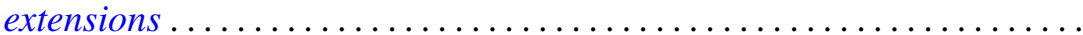

Caulton Lee Irwin, Inverting operators for singular boundary value

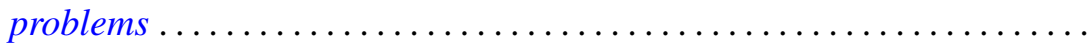

Abraham A. Klein, Matrix rings of finite degree of nilpotency ............ 387

Wei-Eihn Kuan, On the hyperplane section through a rational point of an algebraic variety...

John Hathway Lindsey, II, On a six-dimensional projective representation of $\mathrm{PSU}_{4}(3)$

Jorge Martinez, Approximation by archimedean lattice cones ...

J. F. McClendon, On stable fiber space obstructions .........

Mitsuru Nakai and Leo Sario, Behavior of Green lines at the Kuramochi boundary of a Riemann surface ....................

Donald Steven Passman, Linear identities in group rings. I. .

Donald Steven Passman, Linear identities in group rings. II ...

David S. Promislow, The Kakutani theorem for tensor products of

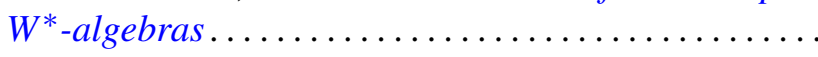

Richard Lewis Roth, On the conjugating representation of a finite group

Bert Alan Taylor, On weighted polynomial approximation of entire functions...

William Charles Waterhouse, Divisor classes in pseudo Galois

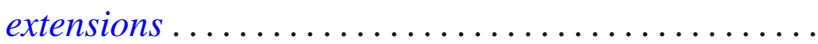

Chi Song Wong, Subadditive functions ...

Ta-Sun $\mathrm{Wu}$, A note on the minimality of certain bitransformation groups 